\title{
Microstructure and Mechanical Properties Evolution in HfNbTaTiZr Refractory High-Entropy Alloy During Cold Rolling
}

\author{
Sergey Zherebtsov, Nikita Yurchenko,* Dmitry Shaysultanov, Mikhail Tikhonovsky, \\ Gennady Salishchev, and Nikita Stepanov
}

The effect of cold rolling on the structure and mechanical properties of the $\mathrm{HfNbTaTiZr}$ refractory high-entropy alloy with a single body-centered cubic (BCC) phase structure is studied. The microhardness evolution during cold rolling to the thickness strain $\varepsilon_{\mathrm{th}}=80 \%$ shows three distinct stages: an increase till $\varepsilon_{\mathrm{th}} \approx 15 \%$, a plato in the interval $\approx 15-40 \%$, and again some increase at $\varepsilon_{\text {th }} \geq 40 \%$. This behavior is found to be associated with an increase in dislocation density at the first stage, the formation of kink bands at the second stage, and the development of shear bands with fine lamellar internal substructure at the third stage. After cold rolling to $80 \%$, the alloy demonstrates the yield strength of $1220 \mathrm{MPa}$, peak strength $1320 \mathrm{MPa}$, and elongation to fracture 3.4\%. A short steady-state flow stage is observed on the engineering stress-strain curve that can also be associated with kinking. belongs to a so-called refractory highentropy-alloy (RHEA) family, which was introduced by Senkov et al. in the framework of the new high-temperature materials development. ${ }^{[6]}$ Usually, RHEAs demonstrate high strength at elevated temperatures and very low ductility at room temperature. ${ }^{[3,7-9]}$ The HfNbTaTiZr RHEA, however, shows a quite different behavior: this alloy is very ductile at room temperature (both in compression and tension) and can be cold rolled to a high strain $(>80 \%$ of thickness strain); ${ }^{[10-12]}$ at the same time, it softens rapidly with an increase in temperature. ${ }^{[5,10,13-16]}$ Both the yield stress and elongation to fracture of this alloy is rather high; however, one of the reported specific features of this RHEA is associated with High entropy alloys (HEAs) are metallic materials containing more than five elements, taken mostly in equiatomic concentrations. ${ }^{[1]}$ Such a composition was supposed to result in the high entropy of mixing, thereby allowing the formation of simple face-centered cubic (FCC), body-centered cubic (BCC), or hexagonal close-packed (HCP) structures without intermetallic phases. ${ }^{[1]}$ In addition, sluggish diffusion, severe lattice distortion, and cocktail effect were expected to occur in this class of alloys. ${ }^{[2]}$ Although the contribution of the high entropy of mixing to phase structure formation was recently established as neither sufficient nor necessary, ${ }^{[2]}$ the HEA concept yielded a few equiatomic alloys with a unique structure-property combination. ${ }^{[3,4]}$

One of the examples of the "model" HEA is an equiatomic HfNbTaTiZr alloy with a single-phase bcc structure. ${ }^{[5]}$ This alloy

Prof. S. Zherebtsov, Dr. N. Yurchenko, Dr. D. Shaysultanov,

Prof. G. Salishchev, Dr. N. Stepanov

Laboratory of Bulk Nanostructured Materials

Belgorod National Research University

Belgorod 308015, Russia

E-mail: yurchenko_nikita@bsu.edu.ru

Prof. M. Tikhonovsky

National Science Center "Kharkov Institute of Physics and Technology"

NAS of Ukraine

Kharkov 61108, Ukraine

The ORCID identification number(s) for the author(s) of this article can be found under https://doi.org/10.1002/adem.202000105.

DOI: 10.1002/adem.202000105
Although the HfNbTaTiZr alloy is the most studied RHEA so far, there is a lack of systematic investigations on the deformation mechanisms of this alloy in different conditions. Couzinié et al. have shown that the main deformation mechanisms of the alloy at ambient temperature were consequently related to the movement of screw dislocations, localization of deformation in bands, and further formation of dislocation dipoles, loops, and tangles. ${ }^{[17]}$ Later, the same authors observed the formation of shear bands and kink bands during compression of the alloy at different strain rates. ${ }^{[18]}$ However, the evolution of the microstructure and properties of this RHEA during such an industrially important deformation scheme as cold rolling is still an open issue. ${ }^{[10-12]}$

In this study, the microstructure evolution of the HfNbTaTiZr RHEA during cold rolling, as well as mechanical properties of the severely deformed alloy, was thoroughly examined, and a crucial role of deformation kinking in an unusual mechanical behavior (low work hardening) of this alloy was revealed.

In the initial state, the HfNbTaTiZr alloy had a wellrecrystallized single BCC phase microstructure with an average grain size of $200 \pm 90 \mu \mathrm{m}$ (not shown). Rolling to $\varepsilon_{\text {th }}=15 \%$ resulted in the development of a substructure and the formation of numerous relatively thin $(\approx 3-5 \mu \mathrm{m})$ lens-shaped kink bands (Figure 1a, b). The misorientation of kink band boundaries varied from $\approx 20^{\circ}$ (high-angle misorientation) in the vicinity of the initial grain boundaries (misorientation profile \#1 in Figure 1b) to $\approx 5^{\circ}-10^{\circ}$ (low-angle misorientation) at some distance apart from the grain boundaries (misorientation profile \#2 in Figure 1b). a very low work-hardening rate during deformation. ${ }^{[10,14-16]}$ 

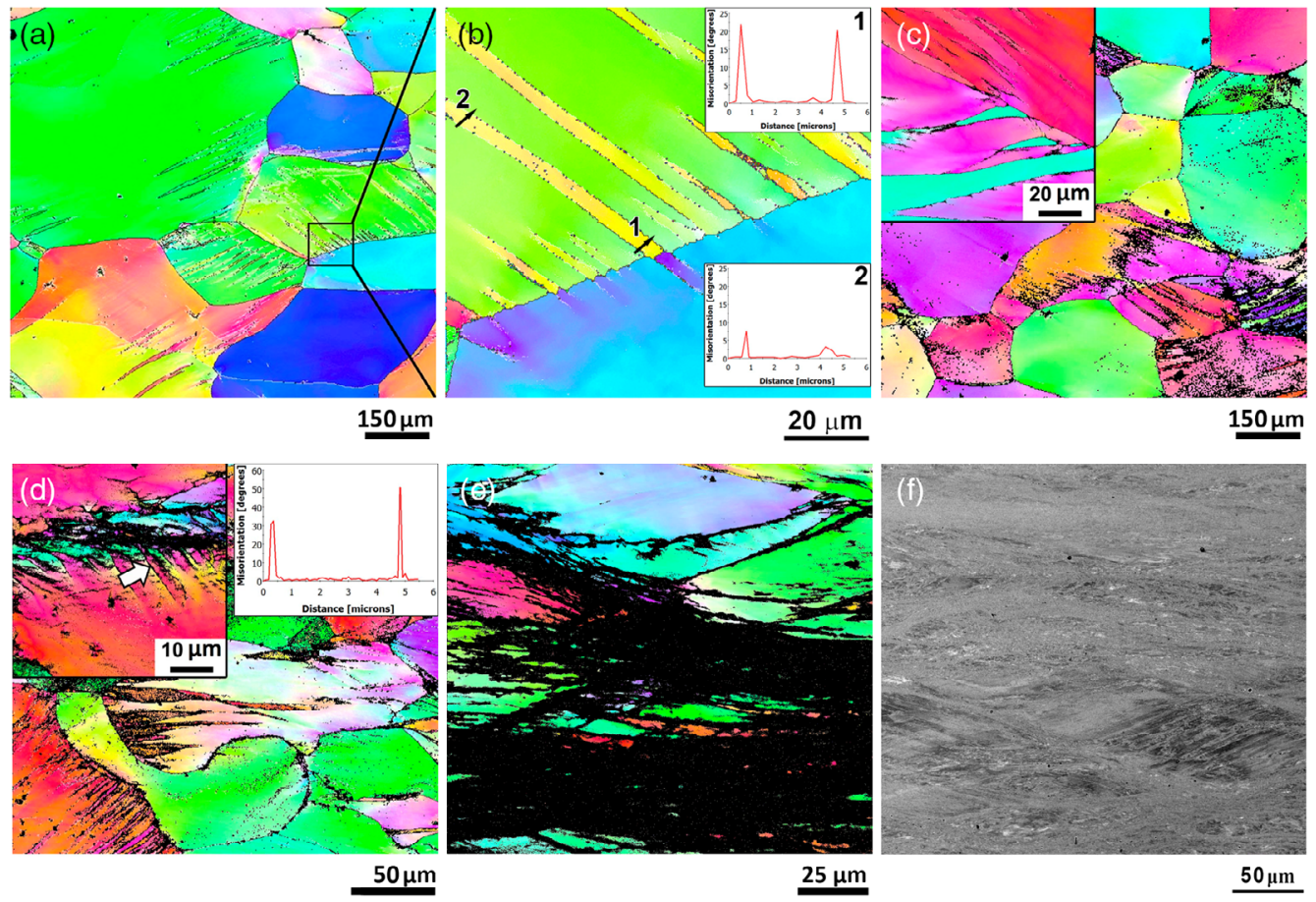

Figure 1. Microstructure of the HfNbTaTiZr alloy after cold rolling to $\left.\epsilon_{\text {th }}=a, b\right) 15 \%$, c) $25 \%$, d) $40 \%$, e) $60 \%$, f) $80 \%$; a-e) EBSD IPF maps; f) SEM-BSE image. The rolling direction is horizontal in all cases.

With an increase in $\varepsilon_{\text {th }}$ to $25 \%$ and $40 \%$, the kink bands tended to become wider (Figure 1c,d). In addition, the misorientation of boundaries of the kink bands increased to $\approx 30-50^{\circ}$ at $\varepsilon_{\mathrm{th}}=40 \%$ (Figure $1 \mathrm{~d}$ ). It should be noted, however, that in the interval of strain from $15 \%$ to $40 \%$, there was no observed noticeable increase in the density of kink bands; that is quite similar to the deformation twinning exhausting in BCC beta titanium alloys or HCP alpha titanium with an increase in plastic strain. ${ }^{[19,20]}$

At $\varepsilon_{\text {th }}=60 \%$, strain localization due to shear bands formation was observed; meanwhile, the microstructure of the adjacent regions was found to be almost intact (Figure 1e). An increase in strain resulted in the involvement of the whole microstructure into shear deformation so that after rolling to $\varepsilon_{\text {th }}=80 \%$ the EBSD map (not shown) comprised a very small fraction of dots with a high-enough confidence index (i.e., $\mathrm{CI} \geq 0.1$ ). Scanning electron microscope (SEM)-backscattered electron (BSE) image shows a typical largely deformed microstructure composed of elongated grains subdivided by multiple shear/kink bands (Figure 1f).

For a better understanding of the microstructure evolution of the HfNbTaTiZr alloy during cold rolling, a transmission electron microscopy (TEM) investigation was conducted (Figure 2). Deformation to $\varepsilon_{\text {th }}=15 \%$ resulted in a considerable increase in dislocation density; dislocations were distributed quite heterogeneously in the microstructure, however. The substructure mainly consisted of screw dislocations, which often formed dislocation bands or elongated pile-ups (Figure 2a), whereas the regions between the dislocation pile-ups contained only some individual dislocations. An increase in strain intensified dislocation slip, thereby increasing dislocation density between the dislocation bands. After $\varepsilon_{\mathrm{th}}=25 \%$, relatively straight thin boundaries with moderate misorientations $\left(7-15^{\circ}\right)$, separating areas with different dislocation densities, were observed in the microstructure (Figure 2b). Most likely, these boundaries are the boundaries of the kink bands observed in EBSD maps (Figure 1).

Localized shear bands $(0.5-1.0 \mu \mathrm{m}$ width) were observed to form after $\varepsilon_{\mathrm{th}}=40 \%$; they contained fine lamellar structure with mainly low-angle misorientation between the lamellae (Figure 2c). The thickness of the lamellae was found to be $\approx 30 \mathrm{~nm}$. The lamellae were divided by transversal (sub)grain boundaries and contained a high density of dislocations (Figure 2d). After cold rolling to the maximum strain $\left(\varepsilon_{\mathrm{th}}=80 \%\right)$, the fraction of the lamellar bands increased, resulting in microstructure refinement. However, the microstructure remained quite heterogeneous, consisting of areas with a very high dislocation density and shear bands with internal fine lamellar microstructures.

Microhardness measurements and tensile tests were used to evaluate the mechanical properties of the alloy. Figure 3a shows the engineering stress-strain curve obtained during uniaxial tension at room temperature of the HfNbTaTiZr alloy subjected to $\varepsilon_{\mathrm{th}}=80 \%$ cold rolling. The alloy, after attaining the yield point at $1220 \mathrm{MPa}$, shows a short strain hardening stage followed by a steady-state flow stage. The peak strength of the largely deformed alloy was $1320 \mathrm{MPa}$; elongation to fracture was $3.4 \%$. The obtained mechanical properties are similar to those reported earlier by Senkov and Semiatin (yield stress and peak strength was 1202 and $1295 \mathrm{MPa}$, respectively). ${ }^{[10]}$

Figure 3a shows the microhardness evolution during cold rolling. Rolling to a relatively low strain of $15 \%$ resulted in a rapid increase in the microhardness from 323 to $364 \mathrm{HV}$. Further strain in the interval of $15-40 \%$ did not result in notable changes 

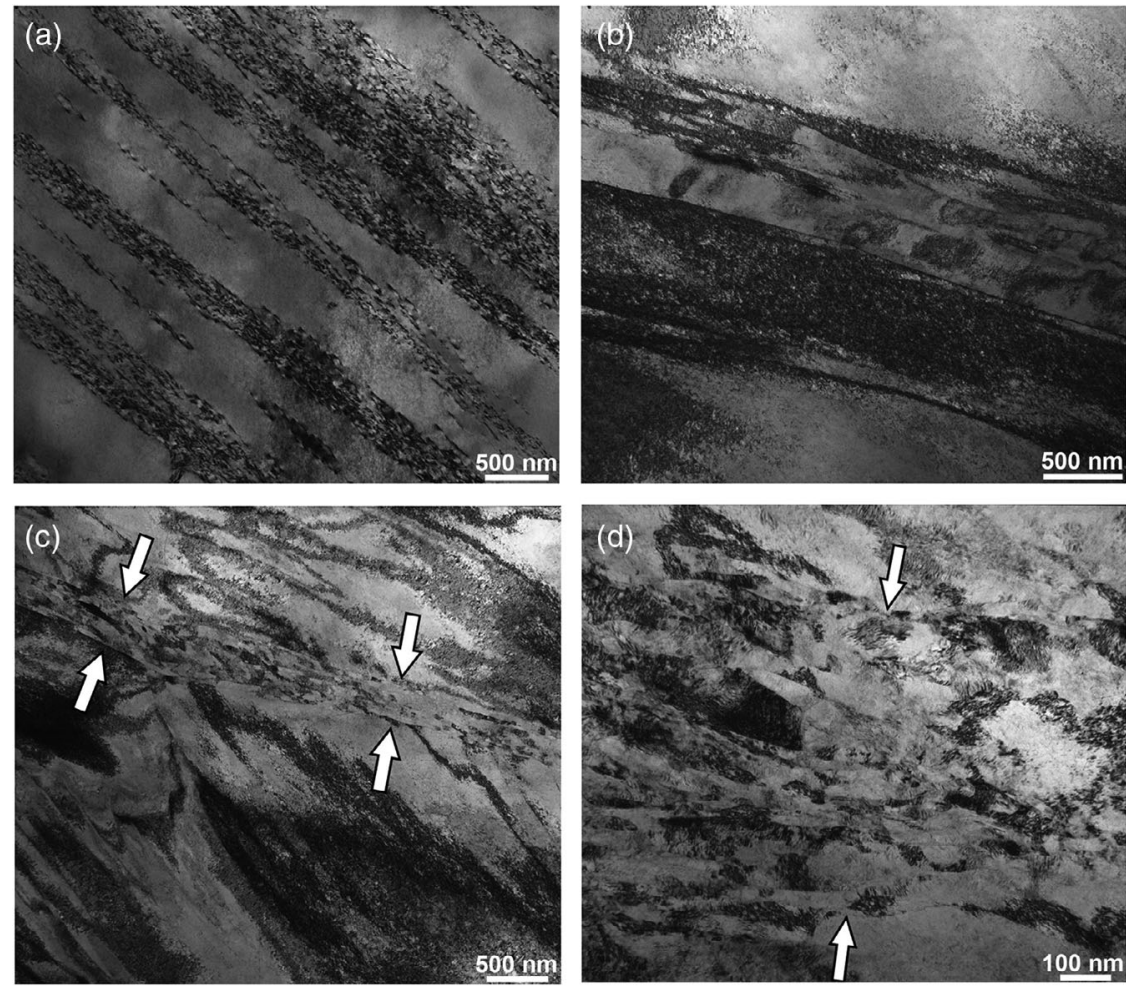

Figure 2. TEM bright-field images of the microstructure of the HfNbTaTiZr alloy rolled to thickness strain of a) $15 \%$, b) $25 \%$, c) $40 \%$, and d) $80 \%$.
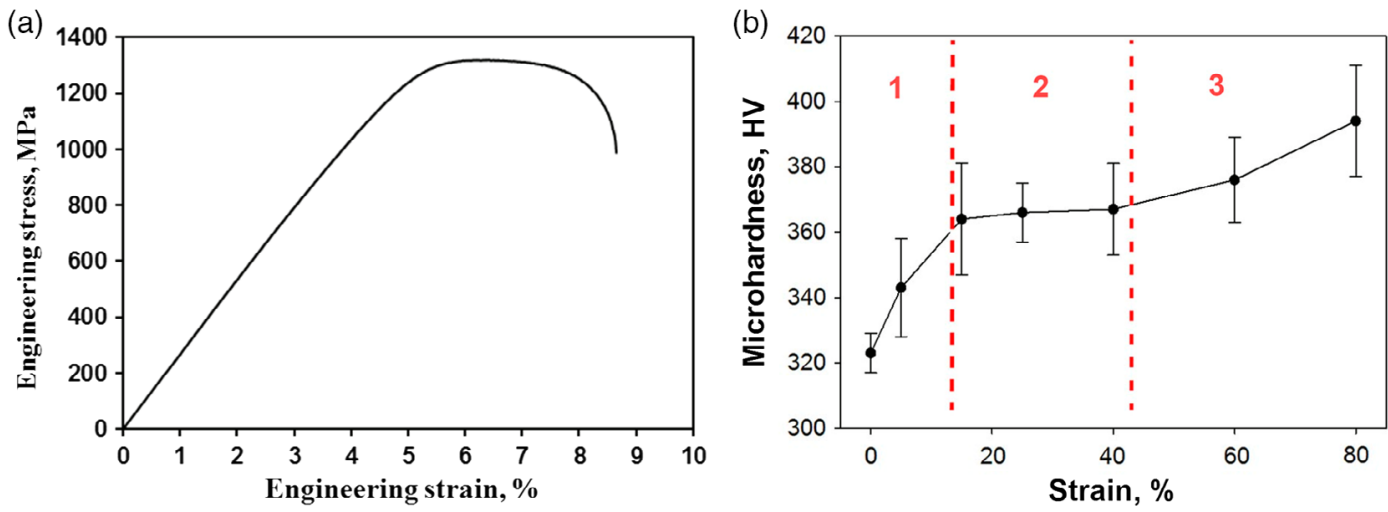

Figure 3. Mechanical properties of the HfNbTaTiZr alloy: a) tensile engineering stress-strain curve after $\epsilon_{\text {th }}=80 \%$ and b) microhardness as a function of strain during cold rolling.

in the microhardness. However, some increase in the microhardness to $394 \mathrm{HV}$ was observed during further rolling to $\varepsilon_{\mathrm{th}}=80 \%$.

The presented results have revealed complex changes in microstructure and mechanical properties of the HfNbTaTiZr alloy during cold rolling. The initial stage of rolling $\left(\varepsilon_{\mathrm{th}} \leq 15 \%\right)$ was mostly associated with the dislocation substructure formation (Figure 2a,b); the observed increase in the dislocation density resulted in substantial hardening (stage 1 in Figure $3 \mathrm{~b}$ ).

An increase in strain to $\varepsilon_{\text {th }} \approx 15-40 \%$ led to the extensive kink bands development (Figure 1a-d, and 2b). Kink bands were first reported by Orowan; ${ }^{[21]}$ later, they were examined in detail by many other researchers. ${ }^{[22,23]}$ Kinking was observed in some alloys with BCC, HCP, and FCC structures, including refractory HEAs with BCC structure. ${ }^{[18,22,24-29]}$ As kinking can be considered as a type of cooperative deformation mechanism being controlled by slip, ${ }^{[23]}$ the increase in dislocation density at the first stage (Figure $3 \mathrm{~b}$ ) induced the activation of kinking. In turn, the kink band formation improved the deformability of the alloy by stress relaxation and crystal reorientation, leading to geometric softening ${ }^{[30]}$ observed at the second stage. The prolonged steadystate flow stage was reported in the $\mathrm{Cu}-\mathrm{Nb}$ nanocomposite that was deformed by kinking. ${ }^{[31]}$ In a Ti-22.4Nb-0.73Ta-2Zr-1.34O gum alloy and other beta titanium alloys, ${ }^{[28,32]}$ kinking was even accompanied by some softening. Thus, the development of kink 
bands in our case can also be a reason for the low work hardening of the alloy during cold rolling in the interval $\varepsilon_{\mathrm{th}} \approx 15-40 \%$ (Figure 3a).

Kinking terminated during further rolling $\left(\varepsilon_{\mathrm{th}}>40 \%\right)$ due to the development of another deformation mechanism, which is associated with the shear bands formation (Figure 2c,d). Shear banding is widespread phenomena in heavily deformed metals and alloys; in the investigated alloy the propagation of shear bands with the developed internal substructure resulted in a very pronounced refinement of the microstructure. An increase in the microhardness at the third stage (Figure 3b) can be ascribed therefore to the Hall-Petch strengthening due to considerable microstructure refinement.

Therefore, the observed increase in microhardness at the first $\left(\varepsilon_{\mathrm{th}} \leq 15 \%\right)$ or third $\left(\varepsilon_{\mathrm{th}} \geq 40 \%\right)$ stages of cold rolling can be associated with an increased dislocation density or the formation of shear bands, respectively, whereas the low work hardening at the second stage $\left(15 \% \leq \varepsilon_{\text {th }} \leq 40 \%\right)$ can be caused by crystal reorientation and geometric softening due to kinking. The kink band formation in the areas not involved in shear deformation during rolling was most likely responsible also for the steady-state stage during tension (Figure 3a). However, this point, as well as the influence of the chemical composition of RHEAs ${ }^{[24]}$ on kinking, still needs further investigations.

The effect of cold rolling on the structure and mechanical properties of the HfNbTaTiZr RHEA was studied. The following conclusions were made: 1) Cold rolling to $80 \%$ of thickness reduction resulted in three distinct stages at the microhardnessthickness strain dependence. Deformation behavior was associated with hardness increasing till $\varepsilon_{\mathrm{th}} \approx 15 \%$, very limited variation in the interval $15-40 \%$, and then some increase at $\varepsilon_{\text {th }} \geq 40 \%$. 2) Analysis of microstructure evolution suggested that these stages were associated with an increase in dislocation density at the first stage, the formation of kink bands at the second stage, and the development of shear bands with a fine lamellar internal substructure at the third stage. 3) The cold rolled to $\varepsilon_{\text {th }}=80 \%$ alloy demonstrated a yield strength value of $1220 \mathrm{MPa}$, peak strength $=1320 \mathrm{MPa}$, and elongation to fracture of $3.4 \%$. The tensile curve had a short steady-state flow stage, which can be associated with the formation of kink bands.

\section{Experimental Section}

The HfNbTaTiZr alloy was produced by vacuum arc melting of pure ( $\geq 99.9 \mathrm{wt} \%$ ) elements, constituting the alloy, in a high purity argon atmosphere. The size of the produced ingot was $\approx 10 \times 14 \times 50 \mathrm{~mm}^{3}$. Rectangular samples measured $8 \times 10 \times 20 \mathrm{~mm}^{3}$ were cut by an electric discharge machine from the as-cast ingot and annealed at $1200^{\circ} \mathrm{C}$ for $24 \mathrm{~h}$; hereafter, this condition will be referred to as the initial one. Unidirectional multipass rolling at room temperature using a fixed rolling speed of $30 \mathrm{~mm} \mathrm{~s}^{-1}$ to a thickness strain $\left(\varepsilon_{\mathrm{th}}\right)$ of $5,15,25,40,60$, and $80 \%$ was conducted in air using a reduction per pass of $\approx 0.1-0.2 \mathrm{~mm}$.

The microstructure of the alloy was examined using SEM, electron backscatter diffraction (EBSD) analysis, and TEM. EBSD was conducted in a FEI Nova NanoSEM 450 field emission-gun scanning electron microscope (FEG SEM) equipped with a Hikari EBSD detector and a TSL OIM system version 6.0. On the inverse pole figure (IPF) maps, high- and lowangle boundaries were indicated with black and white lines, respectively. Points with low confidence index $(\mathrm{Cl} \leq 0.1)$ were excluded from analysis and are shown as black dots. TEM examinations were conducted using a
JEOL JEM-2100 microscope in a midthickness of the rolled specimens. Misorientations across the (sub)grain boundaries were determined using the conventional TEM Kikuchi line method ${ }^{[33]}$ with a technique of converged beam.

Vickers microhardness was examined using a load of $0.2 \mathrm{~kg}$. Specimens with the gauge measured $6 \times 3 \times 1 \mathrm{~mm}^{3}$ were used for mechanical tests in tension at a constant crosshead speed of $10^{-3} \mathrm{~s}^{-1}$ to fracture.

\section{Acknowledgements}

The authors gratefully acknowledge the financial support from the Russian Science Foundation grant no. 19-79-30066. The authors are grateful to the personnel of the Joint Research Center, "Technology and Materials," Belgorod National Research University, for their assistance.

\section{Conflict of Interest}

The authors declare no conflict of interest.

\section{Keywords}

cold rolling, deformation kinking, microstructures, properties, refractory high entropy alloys

Received: January 28, 2020

Revised: March 13, 2020

Published online: April 28, 2020

[1] J. W. Yeh, S. K. Chen, S. J. Lin, J. Y. Gan, T. S. Chin, T. T. Shun, C. H. Tsau, S. Y. Chang, Adv. Eng. Mater. 2004, 6, 299.

[2] D. B. Miracle, O. N. Senkov, Acta Mater. 2017, 122, 448.

[3] O. N. Senkov, G. B. Wilks, J. M. Scott, D. B. Miracle, Intermetallics 2011, 19, 698

[4] M. Biesuz, T. G. Saunders, K. Chen, M. Bortolotti, M. Salvo, S. Grasso, M. J. Reece, J. Eur. Ceram. Soc. 2019.

[5] O. N. Senkov, J. M. Scott, S. V. Senkova, D. B. Miracle, C. F. Woodward, J. Alloys Compd. 2011, 509, 6043.

[6] O. N. Senkov, G. B. Wilks, D. B. Miracle, C. P. Chuang, P. K. Liaw, Intermetallics 2010, 18, 1758.

[7] O. N. Senkov, S. V. Senkova, D. B. Miracle, C. Woodward, Mater. Sci. Eng. A 2013, 565, 51.

[8] N. D. Stepanov, N. Y. Yurchenko, D. V. Skibin, M. A. Tikhonovsky, G. A. Salishchev, J. Alloys Compd. 2015, 652, 266.

[9] N. Y. Yurchenko, N. D. Stepanov, D. G. Shaysultanov, M. A. Tikhonovsky, G. A. Salishchev, Mater. Charact. 2016, 121, 125.

[10] O. N. Senkov, S. L. Semiatin, J. Alloys Compd. 2015, 649, 1110.

[11] W. Wu, S. Ni, Y. Liu, M. Song, J. Mater. Res. 2016, 31, 3815.

[12] R. R. Eleti, V. Raju, M. Veerasham, S. R. Reddy, P. P. Bhattacharjee, Mater. Charact. 2018, 136, 286.

[13] O. N. Senkov, J. M. Scott, S. V. Senkova, F. Meisenkothen, D. B. Miracle, C. F. Woodward, J. Mater. Sci. 2012, 47, 4062.

[14] O. N. Senkov, A. L. Pilchak, S. L. Semiatin, Metall. Mater. Trans. A 2018, 49, 2876.

[15] C. C. Juan, M. H. Tsai, C. W. Tsai, W. L. Hsu, C. M. Lin, S. K. Chen, S. J. Lin, J. W. Yeh, Mater. Lett. 2016, 184, 200.

[16] S. Chen, K. K. Tseng, Y. Tong, W. Li, C. W. Tsai, J. W. Yeh, P. K. Liaw, J. Alloys Compd. 2019, 795, 19.

[17] J.-P. Couzinié, L. Lilensten, Y. Champion, G. Dirras, L. Perrière, I. Guillot, Mater. Sci. Eng. A 2015, 645, 255. 
[18] G. Dirras, H. Couque, L. Lilensten, A. Heczel, D. Tingaud, J. P. Couzinié, L. Perrière, J. Gubicza, I. Guillot, Mater. Charact. 2016, 111, 106.

[19] G. H. Zhao, X. Xu, D. Dye, P. E. J. Rivera-Díaz-del-Castillo, Acta Mater. 2020, 183, 155.

[20] S. V. Zherebtsov, G. S. Dyakonov, G. A. Salishchev, A. A. Salem, S. L. Semiatin, Metall. Mater. Trans. A Phys. Metall. Mater. Sci. 2016, 47, 5101.

[21] E. Orowan, Nature 1942, 149, 643.

[22] C. S. Hess, J. B. Barnett, Trans. Met. Soc. A.I.M.E. 1949, 185, 599.

[23] A. G. Crocker, J. S. Abell, Philos. Mag. 1976, 33, 305.

[24] S. Wang, M. Wu, D. Shu, B. Sun, Mater. Lett. 2020, 264, 127369.

[25] N. Y. Yurchenko, E. S. Panina, S. V. Zherebtsov, M. A. Tikhonovsky, G. A. Salishchev, N. D. Stepanov, Mater. Charact. 2019, 158, 109980.

[26] T. Sakai, H. Miura, X. Yang, Mater. Sci. Eng. A 2009, 499, 2.
[27] S. Sadeghpour, S. M. Abbasi, M. Morakabati, L. P. Karjalainen, D. A. Porter, Mater. Sci. Eng. A 2018, 731, 465.

[28] S. Sadeghpour, S. M. Abbasi, M. Morakabati, L. P. Karjalainen, J. Alloys Compd. 2019, 808, 151741.

[29] K. Hagihara, T. Mayama, M. Honnami, M. Yamasaki, H. Izuno, T. Okamoto, T. Ohashi, T. Nakano, Y. Kawamura, Int. J. Plast. 2016, 77, 174.

[30] A. T. Churchman, Acta Metall. 1955, 3, 22.

[31] T. Nizolek, N. A. Mara, I. J. Beyerlein, J. T. Avallone, T. M. Pollock, Adv. Eng. Mater. 2015, 17, 781.

[32] Y. Yang, S. Q. Wu, G. P. Li, Y. L. Li, Y. F. Lu, K. Yang, P. Ge, Acta Mater. 2010, 58, 2778

[33] D. B. Williams, C. B. Carter, Transmission Electron Microscopy A Textbook For Materials Science, Springer, New York 2009. 